Review Article

\title{
Recent Advances on Filtering and Control for Nonlinear Stochastic Complex Systems with Incomplete Information: A Survey
}

\author{
Bo Shen, ${ }^{1}$ Zidong Wang, ${ }^{1,2}$ Jinling Liang, ${ }^{3}$ and Yurong Liu ${ }^{4}$ \\ ${ }^{1}$ School of Information Science and Technology, Donghua University, Shanghai 200051, China \\ ${ }^{2}$ Department of Information Systems and Computing, Brunel University, Uxbridge, \\ Middlesex UB8 3PH, UK \\ ${ }^{3}$ Department of Mathematics, Southeast University, Nanjing 210096, China \\ ${ }^{4}$ Department of Mathematics, Yangzhou University, Yangzhou 225002, China
}

Correspondence should be addressed to Zidong Wang, zidong.wang@brunel.ac.uk

Received 16 August 2011; Accepted 28 September 2011

Academic Editor: Zhan Shu

Copyright (C) 2012 Bo Shen et al. This is an open access article distributed under the Creative Commons Attribution License, which permits unrestricted use, distribution, and reproduction in any medium, provided the original work is properly cited.

\begin{abstract}
Some recent advances on the filtering and control problems for nonlinear stochastic complex systems with incomplete information are surveyed. The incomplete information under consideration mainly includes missing measurements, randomly varying sensor delays, signal quantization, sensor saturations, and signal sampling. With such incomplete information, the developments on various filtering and control issues are reviewed in great detail. In particular, the addressed nonlinear stochastic complex systems are so comprehensive that they include conventional nonlinear stochastic systems, different kinds of complex networks, and a large class of sensor networks. The corresponding filtering and control technologies for such nonlinear stochastic complex systems are then discussed. Subsequently, some latest results on the filtering and control problems for the complex systems with incomplete information are given. Finally, conclusions are drawn and several possible future research directions are pointed out.
\end{abstract}

\section{Introduction}

Filtering and control problems have long been a fascinating focus of research attracting constant attention from a variety of engineering areas. In recent years, with the rapid development of the network technology, the study of networked control systems (NCSs) has gradually become an active research area due to the advantages of using networked media in many aspects such as low cost, reduced weight and power requirements, simple installation and maintenance, as well as high reliability. It is well known that the devices in networks are mutually connected via communication cables which are of limited capacity. Therefore, some 
new network-induced phenomena have inevitably emerged in the areas of signal processing and control engineering. These phenomena include, but are not limited to, network-induced time delay, data missing (also called packet dropout or missing measurement), quantization, saturation, and channel fading. Note that these phenomena could drastically deteriorate the performance of the networked filtering or control systems and, as such, the aim of this paper is to deal with the filtering and control problems for nonlinear stochastic complex systems with aforementioned network-induced phenomena. In this paper, the information with respect to the network-induced phenomena is customarily referred to as the incomplete information.

Nowadays, practical engineering systems typically exhibit a great deal of complexity which poses significant challenges for the analysis and synthesis of such systems. Among others, the nonlinearity and stochasticity serve as two of the main sources in reality that have resulted in considerable system complexity and have received recurring research attention. Moreover, due to the unavoidable modeling errors and coupled dynamics, some new interesting phenomena (such as parameter uncertainties and coupling between control nodes) should be taken into account to achieve the desired performance. The complexity sources mentioned above give rise to the urgent necessity for developing new filtering and control technologies for various kinds of complex systems in order to meet the needs of practical engineering. It is not surprising that, in the past few years, the control and filtering problems for complex systems with incomplete information have been extensively investigated by many researchers.

In this paper, we focus mainly on the filtering and control problem for complex systems with incomplete information and aim to give a survey on some recent advances in this area. The incomplete information under consideration includes missing measurements, randomly varying sensor delays, signal quantization, sensor saturations, and signal sampling. The modeling issues are first discussed to reflect the real complexity of the nonlinear stochastic systems. Based on the models established, various filtering and control problems with incomplete information are reviewed in detail. Then, we deal with the complex systems from three aspects, that is, nonlinear stochastic systems, complex networks, and sensor networks. Both theories and techniques for dealing with complex systems are reviewed and, at the same time, some challenging issues for future research are raised. Subsequently, the filtering problems for the stochastic nonlinear complex networks with incomplete information are paid particular attention by summarizing the latest results. Finally, some conclusions are drawn and several possible related research directions are pointed out.

The remainder of this paper is organized as follows. In Section 2, the motivation for addressing the incomplete information is discussed. Section 3 reviews the developments of filtering and control issues for three kinds of complex systems. Section 4 gives latest results on filtering problems for the stochastic nonlinear complex networks with incomplete information. The conclusions and future work are given in Section 5.

\section{Incomplete Information}

Recently, the signal transmission via networked systems has become prevalent and, accordingly, network-induced issues have drawn considerable research interests. These issues mainly include missing measurements (also called packet dropouts), randomly varying sensor delays, signal quantization, sensor saturations, and signal sampling whose mathematical models are listed in Table 1 , where $v_{k}$ is the external disturbance while $w_{k}$ represents both the exogenous random inputs and parameter uncertainty of the system. Let 
Table 1: Mathematical models of incomplete information.

\begin{tabular}{ll}
\hline Types & Mathematical models \\
\hline \multirow{2}{*}{ Missing measurements } & $x_{k+1}=f\left(x_{k}\right)+E_{1} v_{k}+g\left(x_{k}\right) w_{k}, y_{k}=\gamma_{k} h\left(x_{k}\right)+E_{2} v_{k}$, where $\gamma_{k}$ is a \\
& stochastic variable taking value on 1 or 0. \\
\hline \multirow{2}{*}{$\begin{array}{l}\text { Randomly varying sensor } \\
\text { delays }\end{array}$} & $x_{k+1}=f\left(x_{k}\right)+E_{1} v_{k}+g\left(x_{k}\right) w_{k}$, \\
& $\begin{array}{l}y_{k}=\gamma_{k} h\left(x_{k}\right)+\left(1-\gamma_{k}\right) h\left(x_{k-1}\right)+E_{2} v_{k}, \text { where } \gamma_{k} \text { is a stochastic } \\
\text { variable taking value on } 1 \text { or } 0 .\end{array}$ \\
\hline \multirow{2}{*}{ Signal quantization } & $x_{k+1}=f\left(x_{k}\right)+E_{1} v_{k}+g\left(x_{k}\right) w_{k}, y_{k}=q\left(h\left(x_{k}\right)\right)+E_{2} v_{k}$, where $q(\cdot)$ \\
\hline Sensor saturations & is a quantization function. \\
\hline Signal sampling & $x_{k+1}=f\left(x_{k}\right)+E_{1} v_{k}+g\left(x_{k}\right) w_{k}, y_{k}=\operatorname{Sat}\left(h\left(x_{k}\right)\right)+E_{2} v_{k}$, where \\
& Sat $(\cdot)$ is a saturation function. \\
\hline
\end{tabular}

us now discuss the network-induced issues one by one as follows in order to motivate the research problem to be investigated.

\subsection{Missing Measurements}

It is quite common in practice that the measurement output of a discrete-time stochastic system is not consecutive but contains missing observations due to a variety of causes such as sensor temporal failure and network-induced packet loss, see, for example, [1-3]. Therefore, it is not surprising that the filtering problem for systems with missing measurements has recently attracted much attention. For example, a binary switching sequence has been used in [4-6], which can be viewed as a Bernoulli distributed white sequence taking values of 0 and 1, to model the measurement missing phenomena. A Markovian jumping process has been employed in [7] to reflect the measurement missing problem. In [8, 9], the data missing (dropout) rate has been converted into the signal transmission delay that has both the upper and lower bounds. In [10], a model of multiple missing measurements has been presented by using a diagonal matrix to account for the different missing probability for individual sensors. By introducing a certain set of indicator functions, the packet dropouts and random sensor delays have been modeled in a unified way in [11]. The optimal $\mathrm{H}_{2}$ filtering problem for linear systems with multiple packet dropouts has been studied in [12], whereas the optimal $H_{\infty}$ filtering problem has been dealt with in [13] for the same systems. Moreover, the optimal filter design problem has been tackled in [14] for systems with multiple packet dropouts by solving a recursive difference equation (RDE).

\subsection{Randomly Varying Sensor Delays}

In practical applications such as engineering, biological, and economic systems, the measured output may be delayed. Therefore, the problem of filtering with delayed measurements has been attracting considerable research interests, see $[7,15,16]$, for some recent publications, where the time-delay in the measurement is customarily assumed to be deterministic. However, it is quite common in practice that the time-delays occur in a random way, rather than a deterministic way, for a number of engineering applications such as real-time distributed decision-making and multiplexed data communication networks. Hence, there is a great need to develop new filtering approaches for the systems with randomly varying 
delayed measurements, and some efforts have been made in this regard so far. For example, in [17], a linear unbiased state estimation problem has been examined for discrete-time systems with random sensor delays over both finite- and infinite-horizons where the full and reducedorder filters have been designed to achieve specific estimation error covariances. These results have been extended in [18] to the case where parameter uncertainties are taken into account. A robust suboptimal filter design problem has been considered for uncertain discrete time-varying systems with randomly varying sensor delay in [19], where some sufficient conditions have been developed for the filter design such that the upper-bound of the state estimation error variance is minimized. Very recently, in [20], a linear matrix inequality (LMI) approach [21] has been developed to discuss the infinite-horizon $H_{\infty}$ filtering problem for linear discrete-time systems with randomly varying sensor delays.

\subsection{Signal Quantization}

The signal quantization is considered as another source that has significant impact on the achievable performance of the networked systems and, therefore, it is necessary to conduct analysis on the quantizers and understand how much effect the quantization makes on the overall networked systems. In fact, the problem of quantized control for nonnetworked systems has been reported as early as in 1990 [22]. So far, a great number of results have been available in the literature, see for example, [22-27]. In [23], the feedback stabilization problems have been considered for linear time-invariant control systems with saturating quantized measurements. In [27], some general types of quantizers have been developed to solve the problem of feedback stabilization for general nonlinear systems. Recently, a new type of quantizer (called logarithmic quantizer) has attracted considerable research interest. Such a quantizer has proven to be the coarsest one in the problem of quadratic stabilization for discrete-time single-input-single-output linear time-invariant systems using quantized feedback under the assumption that the quantizer is static and time-invariant [25]. Based on that, a number of quantized feedback design problems have been studied in [26] for linear systems, where the major contribution of [26] lies in that many quantized feedback design problems have been found to be equivalent to the well-known robust control problems with sector-bounded uncertainties. Later, the elegant results obtained in [25] have been generalized to the multiple-input-multiple-output systems and to control design with performance constraints. Inspiringly, in recent years, there have appeared some new results on NCSs with the consideration of signal quantization effects. In [28], the network-based guaranteed cost problem has been dealt with for linear systems with state and input quantization by using the method of sector bound uncertainties. Moreover, in [29], the problem of quantized state feedback $H_{\infty}$ stabilization has been addressed for linear timeinvariant systems over data networks with limited network quality-of-service. Following that, the problem of output feedback control for NCSs with limited communication capacity has been investigated in [30], where the packet losses and quantization effects are taken into account simultaneously.

\subsection{Sensor Saturations}

In reality, the obstacles in delivering the high performance promises of traditional filter theories are often due to the physical limitations of system components, of which the most commonly encountered one stems from the saturation that occurs in any actuators, sensors, 
or certain system components. Saturation brings in nonlinear characteristics that can severely restrict the amount of deployable filter scheme. Such a characteristic not only limits the filtering performance that can otherwise be achieved without saturation, it may also lead to undesirable oscillatory behavior or, even worse, instability. Therefore, the control problems for systems under actuator/sensor saturations have attracted considerable research interests (see e.g., [31-37]), and the related filtering problem has also gained some scattered research attention $[38,39]$.

It should be pointed out that, in almost all the relevant literature, the saturation is implicitly assumed to occur already. However, in networked environments such as wireless sensor networks, the sensor saturation itself may be subject to random abrupt changes, for example, random sensor failures leading to intermittent saturation, sensor aging resulting in changeable saturation level, repairs of partial components, changes in the interconnections of subsystems, sudden environment changes, modification of the operating point of a linearized model of a nonlinear systems, and so forth. In other words, the sensor saturations may occur in a probabilistic way and are randomly changeable in terms of their types and/or intensity. Such a phenomenon of sensor saturation, namely, randomly occurring sensor saturation (ROSS), has been largely overlooked in the area.

\subsection{Signal Sampling}

With the rapid development of high-speed computers, modern control systems tend to be controlled by digital controllers, that is, only the samples of the control input signals at discrete time instants will be employed. The traditional approach is to use periodic sampling technique to obtain a discrete-time system for modeling the real plant. However, such a discrete-time model might not capture the intersample behavior of the real system, especially for the case when the sampling period is time-varying. On this account, considerable research efforts have been made on various aspects of sampled-data systems. For example, the $\mathrm{H}_{2}$ optimal and $H_{\infty}$ suboptimal control problems for sampled-data systems have been studied in $[40,41]$ and $[42,43]$, respectively. As for the sampled-data filtering problem, let us mention some representative work here. In [44], the robust $H_{\infty}$ filtering problem has been investigated for a class of systems with parametric uncertainties and unknown time delays under sampled measurements. The nonlinear $H_{\infty}$ filtering problem for sampled-data systems has been considered in [45], where a set of certain continuous and discrete Hamilton-Jacobi equations has been established for the existence of the desired filter. In [46], the performance criterion in terms of the estimation error covariance has been proposed and the corresponding sampled-data filtering problem has been solved. It is worth pointing out that, in $[47,48]$, a new approach to dealing with the sampled-data control problems has been proposed by converting the sampling period into a time-varying but bounded delay, and then the sampled-data $H_{\infty}$ control problem has been investigated by recurring to the $H_{\infty}$ control theory for the time-delay systems. Based on this method, the sampled-data $H_{\infty}$ control and filtering problems have been thoroughly investigated in [49] and [50], respectively, where the stochastic sampling has been taken into account.

\section{Complex Systems}

In this section, we take a look at the theories and technologies for handling the filtering and control problems for the complex systems including nonlinear stochastic systems, complex 
networks, and sensor networks. Afterwards, we point out some challenging issues to be studied.

\subsection{Nonlinear Stochastic Systems}

The nonlinearity and stochasticity are arguably two of the main resources in reality that have resulted in considerable system complexity [40]. In the past few years, nonlinear $H_{\infty}$ filtering and $H_{\infty}$ control have been an active branch within the general research area of nonlinear control problems, and a great deal of results have been available in the literature. For the $H_{\infty}$ control problems, we refer the readers to [51-54] and the references therein. With respect to the $H_{\infty}$ filtering problems, we mention some representative work as follows. In [16], the $H_{\infty}$ filtering problem has been investigated for a class of uncertain stochastic time-delay systems with sector-bounded nonlinearities. The $H_{\infty}$ reduced-order approximation of twodimensional digital filters has been considered in [55], while [56] has designed a full-order $H_{\infty}$ filter for 2D Markovian jump systems. In [45], a nonlinear $H_{\infty}$ filtering problem has been studied for sampled-data systems. In $[57,58]$, the $H_{\infty}$ filtering problem has been considered for systems with constant and time-varying delay, respectively. It should be pointed out that, in all the papers mentioned above, the nonlinearities have been assumed to be bounded by a linearity-like form (e.g., Lipschitz and sector conditions), and the filters have been designed by solving a set of LMIs. With respect to general stochastic systems, the nonlinear $H_{\infty}$ filtering problem has been considered for discrete-time systems in [59], and a great effort has been paid in [60] to investigate the $H_{\infty}$ filtering problem for continuous stochastic systems with a very general form.

\subsection{Complex Networks}

Complex networks are made up of interconnected nodes and are used to describe various systems of real world. Many real world systems can be described by complex networks, such as the World Wide Web, telephone call graphs, neural networks, scientific citation web, and so forth. Since the discoveries of the "small-world" and "scale-free" properties of complex networks $[61,62]$, complex networks become a focus point of research which has attracted increasing attention from various fields of science and engineering. In particular, special attention has been paid to the synchronization problem for dynamical complex networks, in which each node is regarded as a dynamical element [63-65]. It has been shown that the synchronization is ubiquitous in many system models of the natural world, for example, the large-scale and complex networks of chaotic oscillators [66-73], the coupled systems exhibiting spatiotemporal chaos and autowaves [74], and the array of coupled neural networks [75-84].

Recently, the synchronization problem for discrete-time stochastic complex networks has drawn much research attention since it is rather challenging to understand the interaction topology of complex networks because of the discrete and random nature of network topology [85]. On one hand, discrete-time networks could be more suitable to model digitally transmitted signals in many application areas such as image processing, time series analysis, quadratic optimization problems, and system identification. On the other hand, the stochastic disturbances over a real complex network may result from the release of probabilistic causes such as neurotransmitters [86], random phase-coupled oscillators [87], and packet dropouts [88]. A great number of results have been available in the recent literature on the general topic of stochastic synchronization problem for discrete-time complex networks. For example, 
in [89], the synchronization stability problem has been studied for a class of complex dynamical networks with Markovian jumping parameters and mixed time delays.

Although the synchronization problem for discrete-time stochastic complex networks is now attracting an increasing research attention, there are still several open problems deserving further investigation. In a real world, virtually all complex networks are timevarying, that is, all the network parameters are explicitly dependent on time. For example, a major challenge in biological networks is to understand and model, quantitatively, the dynamic topological and functional properties of biological networks. Such time, or condition specific biological circuitries are referred to as time-varying networks or structural nonstationary networks, which are common in biological systems. The synchronization problem for time-varying complex networks has received some scattered research interest, where most literature has focused on time-varying coupling or time-varying delay terms. For example, in [90], a time-varying complex dynamical network model has been introduced and it has been revealed that the synchronization of such a model is completely determined by the innercoupling matrix, the eigenvalues, and the corresponding eigenvectors of the coupling configuration matrix of the network. Very recently, in [91], a class of controlled time-varying complex dynamical networks with similarity has been investigated and a decentralized holographic-structure controller has been designed to stabilize the network asymptotically at its equilibrium states. It should be pointed out that, up to now, the general synchronization results for complex networks with time-varying network parameters have been very few, especially when the networks exhibit both discrete-time and stochastic natures.

Closely associated with the synchronization problem is the so-called state estimation problem for complex networks. For large-scale complex networks, it is quite common that only partial information about the network nodes (states) is accessible from the network outputs. Therefore, in order to make use of key network nodes in practice, it becomes necessary to estimate the network nodes through available measurements. Note that the state estimation problem for neural networks (a special class of complex networks) was first addressed in [92] and has then drawn particular research interests, see, for example, [93, 94], where the networks are deterministic and continuous-time. Recently, the state estimation problem for complex networks has also gained much attention, see [95].

\subsection{Sensor Networks}

Sensor networks have recently received increasing interests due to their extensive application in areas such as information collection, environmental monitoring, industrial automation, and intelligent buildings $[96,97]$. In particular, the distributed filtering or estimation for sensor networks has been an ongoing research issue that attracts increasing attention from researchers in the area. Compared to the single sensor, filter $i$ in a sensor network estimates the system state based not only on the sensor $i$ 's measurement but also on its neighboring sensors' measurements according to the topology of the given sensor network. Such a problem is usually referred to as the distributed filtering or estimation problem. The main difficulty in designing distributed filters lies in how to deal with the complicated coupling between one sensor and its neighboring sensors.

Recently, considerable research efforts have been made with respect to distributed filtering, and some novel distributed filters have been proposed. For example, a distributed estimation algorithm for sensor networks has been proposed in [98], where each node computes its estimate as a weighted sum of its own and its neighbors' measurements and estimates, and the weights are adaptively updated to minimize the variance of the estimation 
error. In [99], diffusion strategies have been suggested and then successfully applied to the distributed Kalman filtering, where nodes communicate with their direct neighbors only and the information is diffused across the network. By using the same diffusion strategies, the distributed Kalman smoother has been designed in [100]. In [101], the notion of distributed bounded consensus filters has been proposed and the convergence analysis has been conducted for the corresponding distributed filters. It has been shown in [101] that, in view of the pinning control approach, only a small fraction of sensors are needed to measure the target information while the whole network can be controlled.

In the past few years, the consensus problems of multiagent networks have stirred a great deal of research interests, and a rich body of research results has been reported in the literature, see, for example, [102-110]. Representatively, in [102], a systematical framework of consensus problem has been proposed, and three kinds of networks including directed networks with fixed topology, directed networks with switching topology, and undirected networks with communication delay and fixed topology have been discussed by using the Lyapunov approach and the frequency domain theory. In [105], the $H_{\infty}$ performance constraint has been introduced to the consensus context, and a distributed robust $H_{\infty}$ consensus controller has been designed for the directed networks of agents with time-delay. The consensus protocol has been extended in [107], where the measurement noises have also been taken into account in constructing the consensus protocol. Comparing to the work mentioned above, in [110], the average consensus problems have been studied for agents with integrator dynamics in presence of communication delays. Recently, the consensus problem has also been studied for designing distributed Kalman filters (DKFs). For example, a distributed filter has been introduced in [111] that allows the nodes of a sensor network to track the average of $n$ sensor measurements using an average consensus-based distributed filter called consensus filter. The DKF algorithm presented in [111] has been modified in [112], where another two novel DKF algorithms have been proposed and the communication complexity as well as packet-loss issues have been discussed. The DKF problem considered in [113] is also based on the average consensus, where the node hierarchy has been used with nodes performing different types of processing and communications. Very recently, the consensus-based overlapping decentralized estimation problem has been dealt with in [114] for systems with missing observations and communication faults.

It is worth mentioning that, in almost all the literature concerning the distributed filtering problems, the filter design algorithm has been mainly based on the traditional Kalman filtering theory. Unfortunately, it is now well known that the robust performance of Kalman filters cannot always be guaranteed since Kalman filters tend to be sensitive to model structure drift $[7,45,57,60,115-118]$. As such, a variety of robust and/or $H_{\infty}$ filtering approaches have been proposed in the literature to improve the robustness of the filters against parameter uncertainties and exogenous disturbances. In this sense, it seems natural to include the robust and/or $H_{\infty}$ performance requirements for the distributed consensus filtering problems, and this deserves deep investigation.

\section{Latest Progress}

Very recently, the filtering problem for the stochastic nonlinear complex systems with incomplete information has been intensively studied and some elegant results have been reported. In this section, we highlight some of the newest work with respect to this topic.

(i) In [119], the $H_{\infty}$ filtering problem has been studied for a general class of nonlinear discrete-time stochastic systems with missing measurements and a filter of very general form 
has been designed such that the filtering process is stochastically stable and the filtering error satisfies $H_{\infty}$ performance constraint for all admissible missing observations and nonzero exogenous disturbances under the zero-initial condition. The existence conditions of the desired filter have been derived in terms of the Hamilton-Jacobi-Isaacs inequalities (HJIs). Then, by using similar analysis techniques, the $H_{\infty}$ filtering problem with randomly varying sensor delays has been considered in [120], and a set of parallel results has been derived.

(ii) In order to describe the phenomena of a nonlinear disturbance appearing in a random way, a notion of the randomly occurring nonlinearity has been introduced in [121, 122]. In [121], a new robust $H_{\infty}$ filtering technique has been developed for the Itô-type discrete time-varying stochastic systems with polytopic uncertainties, quantization effects, and randomly occurring nonlinearities. Then, the robust $H_{\infty}$ finite-horizon filtering problem has been studied in [122] for a class of discrete time-varying stochastic systems with norm-bounded uncertainties, multiple randomly occurred nonlinearities, and successive packet dropouts.

(iii) The $H_{\infty}$ filtering problem has been studied in [123] for a class of nonlinear systems with randomly occurring incomplete information, where the considered incomplete information includes both the sensor saturations and the missing measurements. A new phenomenon of sensor saturation, namely, randomly occurring sensor saturation, has first been put forward in order to better reflect the reality in a networked environment. Then, a novel sensor model has been established to account for both the randomly occurring sensor saturation and missing measurement in a unified representation. Based on this sensor model, a regional $H_{\infty}$ filter with a certain ellipsoid constraint has been designed such that the filtering error dynamics is locally mean-square asymptotically stable and the $H_{\infty}$-norm requirement is satisfied.

(iv) In [124], a new distributed $H_{\infty}$-consensus filtering problem over a finite-horizon has been addressed for sensor networks with multiple missing measurements. The so-called $H_{\infty}$-consensus performance requirement is defined to quantify bounded consensus regarding the filtering errors (agreements) over a finite-horizon. A sufficient condition has first been established in terms of a set of difference linear matrix inequalities (DLMIs) under which the expected $H_{\infty}$-consensus performance constraint is guaranteed. Then, the filter parameters have been explicitly parameterized by means of the solutions to a certain set of DLMIs that can be computed recursively. Subsequently, two kinds of robust distributed $H_{\infty}$-consensus filters have been designed for the systems with norm-bounded uncertainties and polytopic uncertainties.

(v) In [125], the distributed $H_{\infty}$ filtering problem is dealt with for a class of polynomial nonlinear stochastic systems in sensor networks. A Lyapunov function candidate whose entries are polynomials has been adopted and then, a sufficient condition for the existence of a feasible solution to the addressed distributed $H_{\infty}$ filtering problem has been derived in terms of parameter-dependent linear matrix inequalities (PDLMIs). For computational convenience, these PDLMIs have further been converted into a set of sums of squares (SOSs) that can be solved effectively by using the semidefinite programming technique.

(vi) In [126], the problem of distributed $H_{\infty}$ filtering in sensor networks using a stochastic sampled-data approach has been investigated. The signal received by each sensor is sampled by a sampler separately with stochastic sampling periods before it is employed by the corresponding filter. By using the method of converting the sampling periods into bounded time-delays, the design problem of the stochastic sampled-databased distributed $H_{\infty}$ filters amounts to solving the $H_{\infty}$ filtering problem for a class of stochastic nonlinear systems with multiple bounded time-delays. Then, by constructing a new Lyapunov functional and employing both the Gronwall's inequality and the Jenson 
integral inequality, a sufficient condition has been derived to guarantee the $H_{\infty}$ performance as well as the exponential mean-square stability of the resulting filtering error dynamics.

Subsequently, the desired sampled-data-based distributed $H_{\infty}$ filters have been designed in terms of the solution to certain matrix inequalities.

(vii) In [127], new synchronization and state estimation problems have been considered for an array of coupled discrete time-varying stochastic complex networks over a finite-horizon. A novel concept of bounded $H_{\infty}$ synchronization has been proposed to handle the time-varying nature of the complex networks. By utilizing a time-varying realvalued function and the Kronecker product, criteria have been established that ensure the bounded $H_{\infty}$ synchronization in terms of a set of recursive linear matrix inequalities (RLMIs). The bounded $H_{\infty}$ state estimation problem has then been studied for the same complex network, where the purpose is to design a state estimator to estimate the network states through available output measurements such that, over a finite-horizon, the dynamics of the estimation error is guaranteed to be bounded with a given disturbance attenuation level. Again, an RLMI approach has been developed for the state estimation problem.

\section{Conclusions and Future Work}

In this paper, we have surveyed some recent advances on the filtering and control for complex systems with incomplete information. The developments of the incomplete information models have been reviewed and various filtering and control problems based on these incomplete information have been discussed. Then, we have introduced basic theories and methods for dealing with filtering and control problems of complex systems and raised a few challenging issues. Subsequently, we have paid particular attention to the filtering problems of the stochastic nonlinear complex systems with incomplete information and given the latest results. Related topics for the future research work are listed below.

(i) In practical engineering, there still exist many more complex yet important network-induced issues which, however, have not been studied. Therefore, these new phenomena of incomplete information should be paid great attention to, and a unified measurement model accounting for these issues simultaneously also remains to be established.

(ii) The polynomial nonlinear system is one of the most important classes of nonlinear systems. The control and filtering problems for polynomial nonlinear systems with kinds of incomplete information are interesting and deserve further investigation. The analysis and synthesis of polynomial nonlinear controllers and filters for the polynomial nonlinear systems would be a challenging research topic.

(iii) The problems of fault detection and fault tolerant control in the presence of incomplete information are of engineering significance, especially when the system is time varying. Hence, the problems of fault detection and fault tolerant control for time-varying systems with incomplete information over a finite time-horizon would be another interesting topic.

(iv) Note that the incomplete information usually occurs in a random way which makes the considered system stochastic. In this case, the performance objection is only required to be achieved in the desired probability. Therefore, the control and filtering problems for nonlinear stochastic systems with probabilistic performance are of significant engineering importance. 
(v) Applications of the existing theories and methodologies to some practical engineering problems such as the mobile robot navigation would be another one of the future work.

\section{Acknowledgments}

This work was supported in part by the National Natural Science Foundation of China under Grant nos. 61134009, 61104125, 61028008, 61174136, 60974030, and 61074129, the Qing Lan Project of Jiangsu Province of China, the Project sponsored by SRF for ROCS of SEM of China, the Engineering and Physical Sciences Research Council (EPSRC) of the UK under Grant GR/S27658/01, the Royal Society of the UK, and the Alexander von Humboldt Foundation of Germany.

\section{References}

[1] M. Basin, E. Sanchez, and R. Martinez-Zuniga, "Optimal linear filtering for systems with multiple state and observation delays," International Journal of Innovative Computing, Information and Control, vol. 3, no. 5, pp. 1309-1320, 2007.

[2] M. Basin, J. Perez, and D. Calderon-Alvarez, "Optimal filtering for linear systems over polynomial observations," International Journal of Innovative Computing, Information and Control, vol. 4, no. 2, pp. 313-320, 2008.

[3] M. S. Mahmoud, Y. Shi, and H. N. Nounou, "Resilient observer-based control of uncertain timedelay systems," International Journal of Innovative Computing, Information and Control, vol. 3, no. 2, pp. 407-418, 2007.

[4] Z. Wang, D. W. C. Ho, and X. Liu, "Variance-constrained filtering for uncertain stochastic systems with missing measurements," IEEE Transactions on Automatic Control, vol. 48, no. 7, pp. 1254-1258, 2003.

[5] Z. Wang, F. Yang, D. W. C. Ho, and X. Liu, "Robust $H_{\infty}$ filtering for stochastic time-delay systems with missing measurements," IEEE Transactions on Signal Processing, vol. 54, no. 7, pp. 2579-2587, 2006.

[6] B. Sinopoli, L. Schenato, M. Franceschetti, K. Poolla, M. I. Jordan, and S. S. Sastry, "Kalman filtering with intermittent observations," IEEE Transactions on Automatic Control, vol. 49, no. 9, pp. 1453-1464, 2004.

[7] P. Shi, M. Mahmoud, S. K. Nguang, and A. Ismail, "Robust filtering for jumping systems with modedependent delays," Signal Processing, vol. 86, no. 1, pp. 140-152, 2006.

[8] H. Gao and T. Chen, " $H_{\infty}$ estimation for uncertain systems with limited communication capacity," IEEE Transactions on Automatic Control, vol. 52, no. 11, pp. 2070-2084, 2007.

[9] H. Gao, T. Chen, and T. Chai, "Passivity and passification for networked control systems," SIAM Journal on Control and Optimization, vol. 46, no. 4, pp. 1299-1322, 2007.

[10] G. Wei, Z. Wang, and H. Shu, "Robust filtering with stochastic nonlinearities and multiple missing measurements," Automatica, vol. 45, no. 3, pp. 836-841, 2009.

[11] X. He, Z. Wang, and D. Zhou, "Robust $H_{\infty}$ filtering for networked systems with multiple state delays," International Journal of Control, vol. 80, no. 8, pp. 1217-1232, 2007.

[12] M. Sahebsara, T. Chen, and S. L. Shah, "Optimal $H_{2}$ filtering in networked control systems with multiple packet dropout," IEEE Transactions on Automatic Control, vol. 52, no. 8, pp. 1508-1513, 2007.

[13] M. Sahebsara, T. Chen, and S. L. Shah, "Optimal $H_{\infty}$ filtering in networked control systems with multiple packet dropouts," Systems \& Control Letters, vol. 57, no. 9, pp. 696-702, 2008.

[14] S. Sun, L. Xie, and W. Xiao, "Optimal full-order and reduced-order estimators for discrete-time systems with multiple packet dropouts," IEEE Transactions on Signal Processing, vol. 56, no. 8, pp. 4031-4038, 2008.

[15] X. Lu, L. Xie, H. Zhang, and W. Wang, "Robust Kalman filtering for discrete-time systems with measurement delay," IEEE Transactions on Circuits and Systems II, vol. 54, no. 6, pp. 522-526, 2007.

[16] Z. Wang, Y. Liu, and X. Liu, " $H_{\infty}$ filtering for uncertain stochastic time-delay systems with sectorbounded nonlinearities," Automatica, vol. 44, no. 5, pp. 1268-1277, 2008. 
[17] E. Yaz and A. Ray, "Linear unbiased state estimation for random models with sensor delay," in Proceedings of the 35th IEEE Conference on Decision and Control, pp. 47-52, Kobe, Japan, December 1996.

[18] Z. Wang, D. W. C. Ho, and X. Liu, "Robust filtering under randomly varying sensor delay with variance constraints," IEEE Transactions on Circuits and Systems II, vol. 51, no. 6, pp. 320-326, 2004.

[19] F. Yang, Z. Wang, G. Feng, and X. Liu, "Robust filtering with randomly varying sensor delay: the finite-horizon case," IEEE Transactions on Circuits and Systems I, vol. 56, no. 3, pp. 1310-1314, 2009.

[20] S. Zhou and G. Feng, " $H_{\infty}$ filtering for discrete-time systems with randomly varying sensor delays," Automatica, vol. 44, no. 7, pp. 1918-1922, 2008.

[21] S. Xu and J. Lam, "A survey of linear matrix inequality techniques in stability analysis of delay systems," International Journal of Systems Science, vol. 39, no. 12, pp. 1095-1113, 2008.

[22] D. F. Delchamps, "Stabilizing a linear system with quantized state feedback," IEEE Transactions on Automatic Control, vol. 35, no. 8, pp. 916-924, 1990.

[23] R. W. Brockett and D. Liberzon, "Quantized feedback stabilization of linear systems," IEEE Transactions on Automatic Control, vol. 45, no. 7, pp. 1279-1289, 2000.

[24] J.-C. Delvenne, "An optimal quantized feedback strategy for scalar linear systems," IEEE Transactions on Automatic Control, vol. 51, no. 2, pp. 298-303, 2006.

[25] N. Elia and S. K. Mitter, "Stabilization of linear systems with limited information," IEEE Transactions on Automatic Control, vol. 46, no. 9, pp. 1384-1400, 2001.

[26] M. Fu and L. Xie, "The sector bound approach to quantized feedback control," IEEE Transactions on Automatic Control, vol. 50, no. 11, pp. 1698-1711, 2005.

[27] D. Liberzon, "Hybrid feedback stabilization of systems with quantized signals," Automatica, vol. 39, no. 9, pp. 1543-1554, 2003.

[28] D. Yue, C. Peng, and G. Y. Tang, "Guaranteed cost control of linear systems over networks with state and input quantisations," IEE Proceedings Control Theory and Applications, vol. 153, no. 6, pp. 658-664, 2006.

[29] C. Peng and Y.-C. Tian, "Networked $H_{\infty}$ control of linear systems with state quantization," Information Sciences, vol. 177, no. 24, pp. 5763-5774, 2007.

[30] E. Tian, D. Yue, and C. Peng, "Quantized output feedback control for networked control systems," Information Sciences, vol. 178, no. 12, pp. 2734-2749, 2008.

[31] Y. Cao, Z. Lin, and B. M. Chen, "An output feedback $H_{\infty}$ controller design for linear systems subject to sensor nonlinearities," IEEE Transactions on Circuits and Systems I, vol. 50, no. 7, pp. 914-921, 2003.

[32] Y. Cao, Z. Lin, and D. G. Ward, "An antiwindup approach to enlarging domain of attraction for linear systems subject to actuator saturation," IEEE Transactions on Automatic Control, vol. 47, no. 1, pp. 140-145, 2002.

[33] Y. Y. Cao, Z. Lin, and D. G. Ward, " $H_{\infty}$ antiwindup design for linear systems subject to input saturation," Journal of Guidance, Control, and Dynamics, vol. 25, no. 3, pp. 455-463, 2002.

[34] T. Hu, Z. Lin, and B. M. Chen, "Analysis and design for discrete-time linear systems subject to actuator saturation," Systems $\mathcal{E}$ Control Letters, vol. 45, no. 2, pp. 97-112, 2002.

[35] Z. Zuo, D. W. C. Ho, and Y. Wang, "Fault tolerant control for singular systems with actuator saturation and nonlinear perturbation," Automatica, vol. 46, no. 3, pp. 569-576, 2010.

[36] Z. Zuo, J. Wang, and L. Huang, "Output feedback $H_{\infty}$ controller design for linear discrete-time systems with sensor nonlinearities," IEE Proceedings Control Theory and Applications, vol. 152, no. 1, pp. 19-26, 2005.

[37] E. Fridman and M. Dambrine, "Control under quantization, saturation and delay: an LMI approach," Automatica, vol. 45, no. 10, pp. 2258-2264, 2009.

[38] Y. Xiao, Y.-Y. Cao, and Z. Lin, "Robust filtering for discrete-time systems with saturation and its application to transmultiplexers," IEEE Transactions on Signal Processing, vol. 52, no. 5, pp. 1266-1277, 2004.

[39] F. Yang and Y. Li, "Set-membership filtering for systems with sensor saturation," Automatica, vol. 45, no. 8, pp. 1896-1902, 2009.

[40] T. Chen and B. A. Francis, "Linear time-varying $H_{2}$-optimal control of sampled-data systems," Automatica, vol. 27, no. 6, pp. 963-974, 1991.

[41] L. Qiu and T. Chen, "H $\mathrm{H}_{2}$-optimal design of multirate sampled-data systems," IEEE Transactions on Automatic Control, vol. 39, no. 12, pp. 2506-2511, 1994.

[42] T. Chen and L. Qiu, " $H_{\infty}$ design of general multirate sampled-data control systems," Automatica, vol. 30, no. 7, pp. 1139-1152, 1994. 
[43] L. Qiu and T. Chen, "Multirate sampled-data systems: all $H_{\infty}$ suboptimal controllers and the minimum entropy controller," IEEE Transactions on Automatic Control, vol. 44, no. 3, pp. 537-550, 1999.

[44] P. Shi, "Filtering on sampled-data systems with parametric uncertainty," IEEE Transactions on Automatic Control, vol. 43, no. 7, pp. 1022-1027, 1998.

[45] S. K. Nguang and P. Shi, "Nonlinear $H_{\infty}$ filtering of sampled-data systems," Automatica, vol. 36, no. 2, pp. 303-310, 2000.

[46] Z. Wang, B. Huang, and P. Huo, "Sampled-data filtering with error covariance assignment," IEEE Transactions on Signal Processing, vol. 49, no. 3, pp. 666-670, 2001.

[47] E. Fridman, A. Seuret, and J.-P. Richard, "Robust sampled-data stabilization of linear systems: an input delay approach," Automatica, vol. 40, no. 8, pp. 1441-1446, 2004.

[48] E. Fridman, U. Shaked, and V. Suplin, "Input/output delay approach to robust sampled-data $H_{\infty}$ control," Systems \& Control Letters, vol. 54, no. 3, pp. 271-282, 2005.

[49] H. Gao, J. Wu, and P. Shi, "Robust sampled-data $H_{\infty}$ control with stochastic sampling," Automatica, vol. 45, no. 7, pp. 1729-1736, 2009.

[50] J. Wu, X. Chen, and H. Gao, " $H_{\infty}$ filtering with stochastic sampling," Signal Processing, vol. 90, no. 4, pp. 1131-1145, 2010.

[51] J. A. Ball, J. W. Helton, and M. L. Walker, " $H_{\infty}$ control for nonlinear systems with output feedback," IEEE Transactions on Automatic Control, vol. 38, no. 4, pp. 546-559, 1993.

[52] N. Berman and U. Shaked, " $H_{\infty}$ control for discrete-time nonlinear stochastic systems," IEEE Transactions on Automatic Control, vol. 51, no. 6, pp. 1041-1046, 2006.

[53] N. Berman and U. Shaked, "H$H_{\infty}$-like control for nonlinear stochastic systems," Systems E Control Letters, vol. 55, no. 3, pp. 247-257, 2006.

[54] A. J. van der Schaft, " $L_{2}$-gain analysis of nonlinear systems and nonlinear state feedback $H_{\infty}$ control," IEEE Transactions on Automatic Control, vol. 37, no. 6, pp. 770-784, 1992.

[55] C. Du, L. Xie, and Y. C. Soh, " $H_{\infty}$ reduced-order approximation of 2-D digital filters," IEEE Transactions on Circuits and Systems I, vol. 48, no. 6, pp. 688-698, 2001.

[56] L. Wu, P. Shi, H. Gao, and C. Wang, " $H_{\infty}$ filtering for 2D Markovian jump systems," Automatica, vol. 44, no. 7, pp. 1849-1858, 2008.

[57] G. Wei and H. Shu, " $H_{\infty}$ filtering on nonlinear stochastic systems with delay," Chaos, Solitons and Fractals, vol. 33, no. 2, pp. 663-670, 2007.

[58] S. Xu, J. Lam, and X. Mao, "Delay-dependent $H_{\infty}$ control and filtering for uncertain Markovian jump systems with time-varying delays," IEEE Transactions on Circuits and Systems I, vol. 54, no. 9, pp. 2070-2077, 2007.

[59] U. Shaked and N. Berman, " $H_{\infty}$ nonlinear filtering of discrete-time processes," IEEE Transactions on Signal Processing, vol. 43, no. 9, pp. 2205-2209, 1995.

[60] W. Zhang, B.-S. Chen, and C.-S. Tseng, "Robust $H_{\infty}$ filtering for nonlinear stochastic systems," IEEE Transactions on Signal Processing, vol. 53, no. 2, pp. 589-598, 2005.

[61] D. J. Watts and S. H. Strogatz, "Collective dynamics of "small-world" networks," Nature, vol. 393, no. 6684, pp. 440-442, 1998.

[62] A.-L. Barabasi and R. Albert, "Emergence of scaling in random networks," Science, vol. 286, no. 5439, pp. 509-512, 1999.

[63] Z. Duan, G. Chen, and L. Huang, “Disconnected synchronized regions of complex dynamical networks," IEEE Transactions on Automatic Control, vol. 54, no. 4, pp. 845-849, 2009.

[64] Z. Li, Z. Duan, G. Chen, and L. Huang, "Consensus of multiagent systems and synchronization of complex networks: a unified viewpoint," IEEE Transactions on Circuits and Systems I, vol. 57, no. 1, pp. 213-224, 2010.

[65] Z. Zuo, C. Yang, and Y. Wang, "A unified framework of exponential synchronization for complex networks with time-varying delays," Physics Letters A, vol. 374, no. 19-20, pp. 1989-1999, 2010.

[66] X. F. Wang and G. Chen, "Synchronization in small-world dynamical networks," International Journal of Bifurcation and Chaos in Applied Sciences and Engineering, vol. 12, no. 1, pp. 187-192, 2002.

[67] J. Jost and M. P. Joy, "Spectral properties and synchronization in coupled map lattices," Physical Review E, vol. 65, no. 1, p. 016201, 2002.

[68] J. Liang, Z. Wang, Y. Liu, and X. Liu, "Robust synchronization of an array of coupled stochastic discrete-time delayed neural networks," IEEE Transactions on Neural Networks, vol. 19, no. 11, pp. 1910-1921, 2008.

[69] R. Palm, "Synchronization of decentralized multiple-model systems by market-based optimization," IEEE Transactions on Systems, Man, and Cybernetics, Part B, vol. 34, no. 1, pp. 665-672, 2004. 
[70] W. He and J. Cao, "Exponential synchronization of hybrid coupled networks with delayed coupling," IEEE Transactions on Neural Networks, vol. 21, no. 4, pp. 571-583, 2010.

[71] F. O. Souza and R. M. Palhares, "Synchronisation of chaotic delayed artificial neural networks: an $H_{\infty}$ control approach," International Journal of Systems Science, vol. 40, no. 9, pp. 937-944, 2009.

[72] W. Lu and T. Chen, "Synchronization of coupled connected neural networks with delays," IEEE Transactions on Circuits and Systems I, vol. 51, no. 12, pp. 2491-2503, 2004.

[73] W. Lu and T. Chen, "Global synchronization of discrete-time dynamical network with a directed graph," IEEE Transactions on Circuits and Systems II, vol. 54, no. 2, pp. 136-140, 2007.

[74] V. Perez-Munuzuri, V. Perez-Villar, and L. O. Chua, "Autowaves for image processing on a two-dimensional CNN array of excitable nonlinear circuits: flat and wrinkled labyrinths," IEEE Transactions on Circuits and Systems I, vol. 40, no. 3, pp. 174-181, 1993.

[75] L. M. Pecora and T. L. Carroll, "Synchronization in chaotic systems," Physical Review Letters, vol. 64, no. 8, pp. 821-824, 1990.

[76] Z. Li and G. Chen, "Global synchronization and asymptotic stability of complex dynamical networks," IEEE Transactions on Circuits and Systems II, vol. 53, no. 1, pp. 28-33, 2006.

[77] J. Cao and J. Lu, "Adaptive synchronization of neural networks with or without time-varying delay," Chaos. An Interdisciplinary Journal of Nonlinear Science, vol. 16, no. 1, p. article 013133, 2006.

[78] X. Hu and J. Wang, "Design of general projection neural networks for solving monotone linear variational inequalities and linear and quadratic optimization problems," IEEE Transactions on Systems, Man, and Cybernetics, Part B, vol. 37, no. 5, pp. 1414-1421, 2007.

[79] H. R. Karimi and H. Gao, "New delay-dependent exponential $H_{\infty}$ synchronization for uncertain neural networks with mixed time delays," IEEE Transactions on Systems, Man, and Cybernetics, Part B, vol. 40, no. 1, pp. 173-185, 2010.

[80] Z. Fei, H. Gao, and W. X. Zheng, "New synchronization stability of complex networks with an interval time-varying coupling delay," IEEE Transactions on Circuits and Systems II, vol. 56, no. 6, pp. 499-503, 2009.

[81] H. Gao, J. Lam, and G. Chen, "New criteria for synchronization stability of general complex dynamical networks with coupling delays," Physics Letters A, vol. 360, no. 2, pp. 263-273, 2006.

[82] S. Mou, H. Gao, J. Lam, and W. Qiang, "A new criterion of delay-dependent asymptotic stability for Hopfield neural networks with time delay," IEEE Transactions on Neural Networks, vol. 19, no. 3, pp. 532-535, 2008.

[83] P. Li, J. Lam, and Z. Shu, “On the transient and steady-state estimates of interval genetic regulatory networks," IEEE Transactions on Systems, Man, and Cybernetics, Part B, vol. 40, no. 2, pp. 336-349, 2010.

[84] Z. Shu and J. Lam, "Global exponential estimates of stochastic interval neural networks with discrete and distributed delays," Neurocomputing, vol. 71, no. 13-15, pp. 2950-2963, 2008.

[85] Z. Toroczkai, "Complex networks: the challenge of interaction topology," Los Alamos Science, no. 29, pp. 94-109, 2005.

[86] J. Buhmann and K. Schulten, "Influence of noise on the function of a "physiological" neural network," Biological Cybernetics, vol. 56, no. 5-6, pp. 313-327, 1987.

[87] K. Wood, C. Van den Broeck, R. Kawai, and K. Lindenberg, "Continuous and discontinuous phase transitions and partial synchronization in stochastic three-state oscillators," Physical Review E, vol. 76, no. 4, p. 041132, 2007.

[88] Z. Wang, D. W. C. Ho, Y. Liu, and X. Liu, "Robust $H_{\infty}$ control for a class of nonlinear discrete timedelay stochastic systems with missing measurements," Automatica, vol. 45, no. 3, pp. 684-691, 2009.

[89] H. Li and D. Yue, "Synchronization of Markovian jumping stochastic complex networks with distributed time delays and probabilistic interval discrete time-varying delays," Journal of Physics A, vol. 43, no. 10, p. article 105101, 2010.

[90] J. Lü and G. Chen, "A time-varying complex dynamical network model and its controlled synchronization criteria," IEEE Transactions on Automatic Control, vol. 50, no. 6, pp. 841-846, 2005.

[91] W. Zhong, J. D. Stefanovski, G. M. Dimirovski, and J. Zhao, "Decentralized control and synchronization of time-varying complex dynamical network," Kybernetika, vol. 45, no. 1, pp. 151167, 2009.

[92] Z. Wang, D. W. C. Ho, and X. Liu, "State estimation for delayed neural networks," IEEE Transactions on Neural Networks, vol. 16, no. 1, pp. 279-284, 2005.

[93] Y. Liu, Z. Wang, and X. Liu, “Design of exponential state estimators for neural networks with mixed time delays," Physics Letters A, vol. 364, no. 5, pp. 401-412, 2007.

[94] Y. He, Q. G. Wang, M. Wu, and C. Lin, "Delay-dependent state estimation for delayed neural networks," IEEE Transactions on Neural Networks, vol. 17, no. 4, pp. 1077-1081, 2006. 
[95] Y. Liu, Z. Wang, J. Liang, and X. Liu, "Synchronization and state estimation for discrete-time complex networks with distributed delays," IEEE Transactions on Systems, Man, and Cybernetics, Part B, vol. 38, no. 5, pp. 1314-1325, 2008.

[96] B. Lu and V. C. Gungor, "Online and remote motor energy monitoring and fault diagnostics using wireless sensor networks," IEEE Transactions on Industrial Electronics, vol. 56, no. 11, pp. 4651-4659, 2009.

[97] G. Cimatti, R. Rovatti, and G. Setti, "Chaos-based spreading in DS-UWB sensor networks increases available bit rate," IEEE Transactions on Circuits and Systems I, vol. 54, no. 6, pp. 1327-1339, 2007.

[98] A. Speranzon, C. Fischione, K. Johansson, and A. Sangiovanni-Vincentelli, "A distributed minimum variance estimator for sensor networks," IEEE Journal on Selected Areas in Communications, vol. 26, no. 4, pp. 609-621, 2008.

[99] F. S. Cattivelli, C. G. Lopes, and A. H. Sayed, "Diffusion strategies for distributed Kalman filtering: formulation and perfor- mance analysis," in Proceedings of the IAPR Workshop on Cognitive Information, Santorini, Greece, June 2008.

[100] F. S. Cattivelli and A. H. Sayed, "Diffusion mechanisms for fixed-point distributed Kalman smoothing," in Proceedings of the European Signal Processing Conference, pp. 1-4, Lausanne, Switzerland, August 2008.

[101] W. Yu, G. Chen, Z. Wang, and W. Yang, “Distributed consensus filtering in sensor networks," IEEE Transactions on Systems, Man, and Cybernetics, Part B, vol. 39, no. 6, pp. 1568-1577, 2009.

[102] R. Olfati-Saber and R. M. Murray, "Consensus problems in networks of agents with switching topology and time-delays," IEEE Transactions on Automatic Control, vol. 49, no. 9, pp. 1520-1533, 2004.

[103] G. Shi and Y. Hong, "Global target aggregation and state agreement of nonlinear multi-agent systems with switching topologies," Automatica, vol. 45, no. 5, pp. 1165-1175, 2009.

[104] L. Xiao and S. Boyd, "Fast linear iterations for distributed averaging," Systems E Control Letters, vol. 53, no. 1, pp. 65-78, 2004.

[105] P. Lin, Y. Jia, and L. Li, "Distributed robust $H_{\infty}$ consensus control in directed networks of agents with time-delay," Systems E Control Letters, vol. 57, no. 8, pp. 643-653, 2008.

[106] Z. G. Hou, L. Cheng, and M. Tan, "Decentralized robust adaptive control for the multiagent system consensus problem using neural networks," IEEE Transactions on Systems, Man, and Cybernetics, Part B, vol. 39, no. 3, pp. 636-647, 2009.

[107] T. Li and J. F. Zhang, "Mean square average-consensus under measurement noises and fixed topologies: necessary and sufficient conditions," Automatica, vol. 45, no. 8, pp. 1929-1936, 2009.

[108] F. Xiao and L. Wang, "Consensus protocols for discrete-time multi-agent systems with time-varying delays," Automatica, vol. 44, no. 10, pp. 2577-2582, 2008.

[109] Y. G. Sun, L. Wang, and G. Xie, "Average consensus in networks of dynamic agents with switching topologies and multiple time-varying delays," Systems $\mathcal{E}$ Control Letters, vol. 57, no. 2, pp. 175-183, 2008.

[110] P. A. Bliman and G. Ferrari-Trecate, "Average consensus problems in networks of agents with delayed communications," Automatica, vol. 44, no. 8, pp. 1985-1995, 2008.

[111] R. Olfati-Saber and J. S. Shamma, "Consensus filters for sensor networks and distributed sensor fusion," in Proceedings of the 44th IEEE Conference Decision and Control and European Control Conference, pp. 6698-6703, Seville, Spain, December 2005.

[112] R. Olfati-Saber, "Distributed Kalman filtering for sensor networks," in Proceedings of the 46th IEEE Conference on Decision and Control, (CDC'07), pp. 5492-5498, New Orleans, LA, USA, December 2007.

[113] I. D. Schizas, S. I. Roumeliotis, G. B. Giannakis, and A. Ribeiro, "Anytime optimal distributed Kalman filtering and smoothing," in Proceedings of the IEEE 14th WorkShop on Statistical Signal Processing, (SSP '07), pp. 368-372, Madison, Wis, USA, August 2007.

[114] S. S. Stanković, M. S. Stanković, and D. M. Stipanović, "Consensus based overlapping decentralized estimation with missing observations and communication faults," Automatica, vol. 45, no. 6, pp. 1397-1406, 2009.

[115] B. D. O. Anderson and J. B. Moore, Optimal Filtering, Prentice-Hall, Englewood Cliffs, NJ, USA, 1979.

[116] H. Gao, X. Meng, and T. Chen, " $H_{\infty}$ filter design for discrete delay systems: a new parameterdependent approach," International Journal of Control, vol. 82, no. 6, pp. 993-1005, 2009.

[117] H. Gao, Y. Zhao, J. Lam, and K. Chen, " $H_{\infty}$ fuzzy filtering of nonlinear systems with intermittent measurements," IEEE Transactions on Fuzzy Systems, vol. 17, no. 2, pp. 291-300, 2009.

[118] S. K. Nguang and P. Shi, " $H_{\infty}$ filtering design for uncertain nonlinear systems under sampled measurements," International Journal of Systems Science, vol. 32, no. 7, pp. 889-898, 2001. 
[119] B. Shen, Z. Wang, H. Shu, and G. Wei, "On nonlinear $H_{\infty}$ filtering for discrete-time stochastic systems with missing measurements," IEEE Transactions on Automatic Control, vol. 53, no. 9, pp. 2170-2180, 2008.

[120] B. Shen, Z. Wang, H. Shu, and G. Wei, " $H_{\infty}$ filtering for nonlinear discrete-time stochastic systems with randomly varying sensor delays," Automatica, vol. 45, no. 4, pp. 1032-1037, 2009.

[121] B. Shen, Z. Wang, H. Shu, and G. Wei, "Robust $H_{\infty}$ finite-horizon filtering with randomly occurred nonlinearities and quantization effects," Automatica, vol. 46, no. 11, pp. 1743-1751, 2010.

[122] B. Shen, Z. Wang, H. Shu, and G. Wei, " $H_{\infty}$ filtering for uncertain time-varying systems with multiple randomly occurred nonlinearities and successive packet dropouts," International Journal of Robust and Nonlinear Control, vol. 21, no. 14, pp. 1693-1709, 2011.

[123] Z. Wang, B. Shen, and X. Liu, " $H_{\infty}$ filtering with randomly occurring sensor saturations and missing measurements," Automatica. In press.

[124] B. Shen, Z. Wang, and Y. S. Hung, "Distributed $H_{\infty}$-consensus filtering in sensor networks with multiple missing measurements: the finite-horizon case," Automatica, vol. 46, no. 10, pp. 1682-1688, 2010.

[125] B. Shen, Z. Wang, Y. S. Hung, and G. Chesi, “Distributed $H_{\infty}$ filtering for polynomial nonlinear stochastic systems in sensor networks," IEEE Transactions on Industrial Electronics, vol. 58, no. 5, pp. 1971-1979, 2011.

[126] B. Shen, Z. Wang, and X. Liu, "A stochastic sampled-data approach to distributed $H_{\infty}$ filtering in sensor networks," IEEE Transactions on Circuits and Systems I, vol. 58, no. 9, pp. 2237-2246, 2011.

[127] B. Shen, Z. Wang, and X. Liu, "Bounded $H_{\infty}$ synchronization and state estimation for discrete timevarying stochastic complex networks over a finite horizon," IEEE Transactions on Neural Networks, vol. 22, no. 1, pp. 145-157, 2010. 


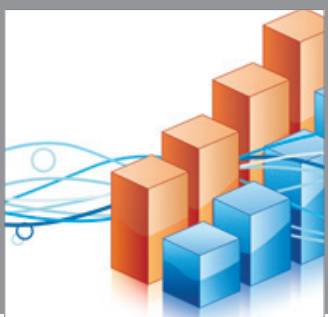

Advances in

Operations Research

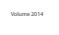

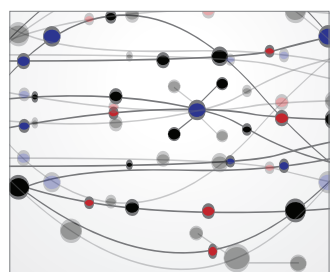

\section{The Scientific} World Journal
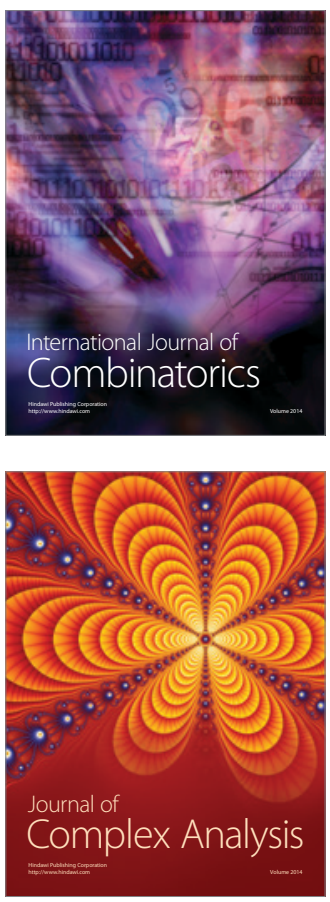

International Journal of

Mathematics and

Mathematical

Sciences
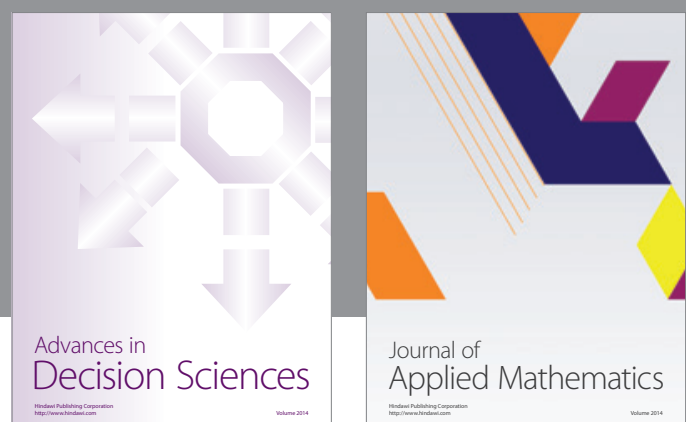

Journal of

Applied Mathematics
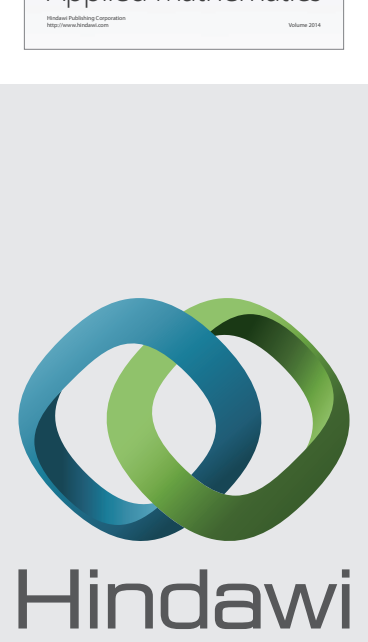

Submit your manuscripts at http://www.hindawi.com
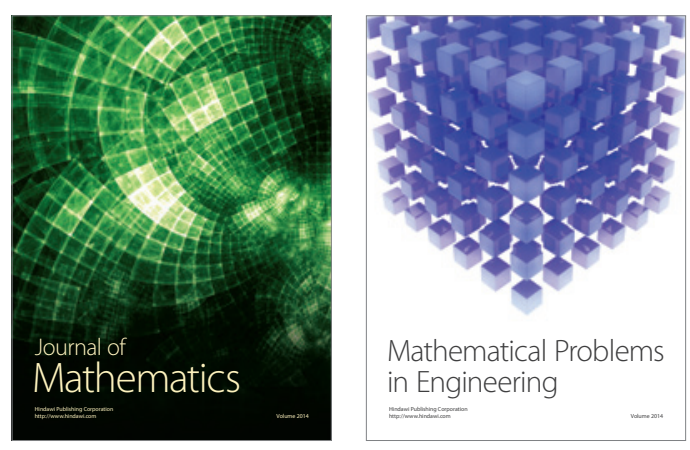

Mathematical Problems in Engineering
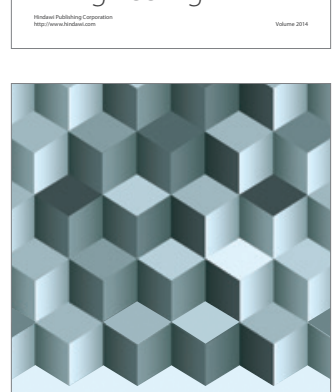

Journal of

Function Spaces
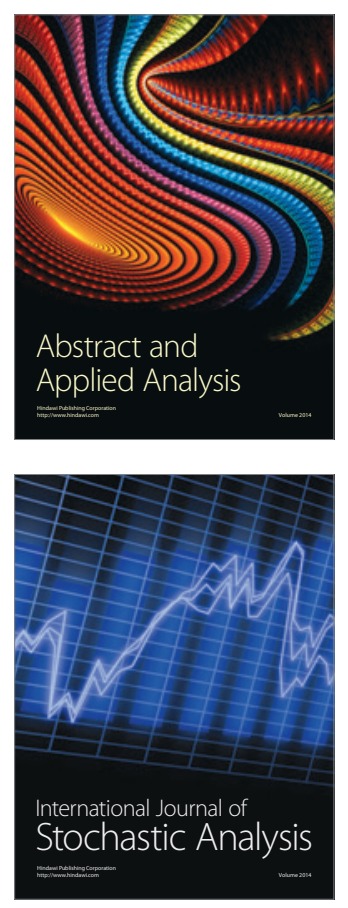

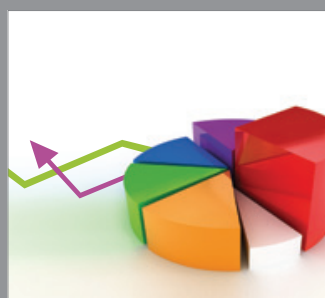

ournal of

Probability and Statistics

Promensencen
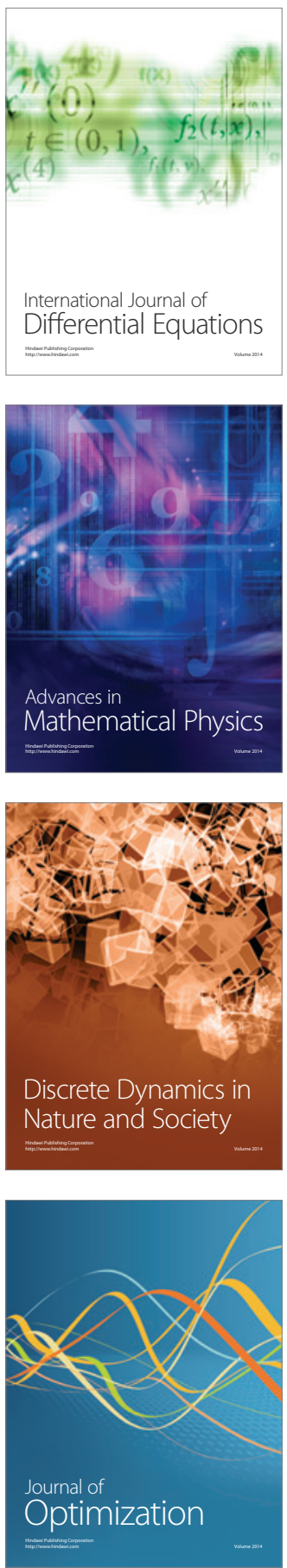